\title{
Harness shared data in international Zika registry
}

\author{
David Baud maternal-fetal medicine specialist ${ }^{1}$, Patrick Gérardin epidemiologist ${ }^{2}$, Audrey Merriam \\ maternal-fetal medicine fellow ${ }^{3}$, Marco P Alves virologist ${ }^{4}$, Didier Musso infectious disease specialist ${ }^{5}$, \\ Blaise Genton tropical medicine specialist ${ }^{6}$, Alice Panchaud teratogen counsellor ${ }^{7}$
}

\begin{abstract}
'Materno-Fetal and Obstetrics Research Unit, Department "Femme-Mère-Enfant," University Hospital, 1011 Lausanne, Switzerland; Institute of Microbiology, Faculty of Biology and Medicine, University of Lausanne and University Hospital, Lausanne, Switzerland; ${ }^{2}$ Pôle Femme Mère Enfant, Centre d'Investigation Clinique (INSERM CIC1410), UM 134 PIMIT "Processus Infectieux en Milieu Insulaire Tropical" (Université de La Réunion, CNRS 9192, INSERM U 1187, IRD 249), CHU Réunion, Saint Pierre, Réunion; ${ }^{3}$ Division of Maternal-Fetal Medicine, Department of Obstetrics and Gynecology, Columbia University Medical Center, New York, New York, USA; ${ }^{4}$ Institute of Virology and Immunology, Department of Infectious Diseases and Pathobiology, University of Bern, Switzerland; ${ }^{5}$ Unit of Emerging Infectious Diseases, Institut Louis Malardé, Tahiti, French Polynesia; ${ }^{6}$ Swiss Tropical and Public Health Institute, Basel, Switzerland; Infectious Disease Service and Department of Ambulatory Care, University Hospital Lausanne, Lausanne, Switzerland; ${ }^{7}$ Swiss Teratogen Information Service, University Hospital, 1011 Lausanne, Switzerland; Division of Clinical Pharmacology, University Hospital, 1011 Lausanne, Switzerland; School of Pharmaceutical Sciences, University of Geneva and Lausanne, Geneva, Switzerland; Department of Epidemiology, Harvard School of Public Health, Boston, MA, USA
\end{abstract}

We welcome the paper by Morris and colleagues on the prevalence of microcephaly in Europe. ${ }^{1}$ The authors state that increases in prevalence such as expected with Zika virus in a non-endemic region would be unlikely to be detected in Europe through routine surveillance.

As a newly identified teratogen, Zika requires global and dedicated tools to allow comprehensive characterisation of the risks associated with its materno-fetal transmission. Nearly a year after the first reported increase in microcephaly in Brazil, ${ }^{2}$ the magnitude of the risks of materno-fetal transmission and the associated potential cofactors remain unclear.

Case reports and congenital anomaly surveillance systems are indispensable tools in detecting new teratogens and have been effective in Brazil's Zika outbreak. However, since these tools will probably not provide global information or allow in-depth comprehension of the link between a teratogen and its risks, we need epidemiological studies, such as case-control studies and cohort studies with appropriate follow-up.

To facilitate these studies we have launched an international web registry, ${ }^{3}$ enrolling pregnant women, prospectively and anonymously, at any gestational age as soon as Zika exposure is suspected (eg, mosquito bite, unprotected sex) and ideally before any additional screening (a prospective registry). Follow-up information on other risk factors and on pregnancy, fetal, and neonatal outcomes is gathered after birth. Any healthcare provider supporting the registry by providing well documented exposures will be regarded as a collaborator of the registry in any future peer reviewed scientific publication (see https://epgl.unige.ch/zika-in-pregnancy-registry/). The collected data will be available to research groups that have a clear, non-redundant research question and approval from a biomedical research ethics committee.

We hope that this centralised dataset of shared information will allow research to progress in acquiring a comprehensive characterisation of the risks associated with Zika materno-fetal transmission.

\section{Competing interests: None declared.}

Full response at: www.bmj.com/content/354/bmj.i4721/rr-0.

1 Morris JK, Rankin J, Garne E, et al. Prevalence of microcephaly in Europe: population based study. BMJ 2016;354:i4721. doi:10.1136/bmj.i4721 pmid:27623840.

Kleber de Oliveira W, Cortez-Escalante J, De Oliveira WT, et al. Increase in reported prevalence of microcephaly in infants born to women living in areas with confirmed Zika virus transmission during the first trimester of pregnancy-Brazil, 2015. MMWR Morb Mortal Wkly Rep 2016;65:242-7. doi:10.15585/mmwr.mm6509e2 pmid:26963593.

3 Panchaud A, Vouga M, Musso D, Baud D. An international registry for women exposed to Zika virus during pregnancy: time for answers. Lancet Infect Dis 2016;16:995-6doi:10. 1016/S1473-3099(16)30255-9.

Published by the BMJ Publishing Group Limited. For permission to use (where not already granted under a licence) please go to http://group.bmj.com/group/rights-licensing/ permissions 